\title{
Dating star clusters in the Small Magellanic Cloud by means of integrated spectra ${ }^{\star}$
}

\author{
A. V. Ahumada ${ }^{1}$, J. J. Clariá ${ }^{1}$, E. Bica ${ }^{2}$, and C. M. Dutra ${ }^{3}$ \\ 1 Observatorio Astronómico, Universidad Nacional de Córdoba, Laprida 854, 5000 Córdoba, Argentina \\ e-mail: andrea@mail.oac.uncor.edu, claria@mail.oac.uncor.edu \\ 2 Instituto de Física, Universidade Federal do Rio Grande do Sul, Av. Bento Gonçalves 9500, Caixa Postal 15051, \\ CEP 91501-970, Porto Alegre RS, Brazil \\ e-mail: bica@if.ufrgs.br \\ 3 Instituto Astronomico e Geofisico da USP, CP 3386, São Paulo 01060-970, SP, Brazil \\ e-mail: dutra@andromeda.iagusp.usp.br
}

Received 4 June 2002 / Accepted 19 July 2002

\begin{abstract}
In this study flux-calibrated integrated spectra in the range (3600-6800) $\AA$ are presented for 16 concentrated star clusters in the Small Magellanic Cloud (SMC), approximately half of which constitute unstudied objects. We have estimated ages and foreground interstellar reddening values from the comparison of the line strengths and continuum distribution of the cluster spectra with those of template cluster spectra with known parameters. Most of the sample clusters are young blue clusters (6-50 Myr), while L 28, NGC 643 and L 114 are found to be intermediate-age clusters (1-6 Gyr). One well known SMC cluster (NGC 416) was observed for comparison purposes. The sample includes clusters in the surroundings and main body of the SMC, and the derived foreground reddening values are in the range $0.00 \leq E(B-V) \leq 0.15$. The present data also make up a cluster spectral library at SMC metallicity.
\end{abstract}

Key words. methods: observational - techniques: spectroscopic - galaxies: Small Magellanic Cloud, star clusters

\section{Introduction}

The study of extragalactic stellar systems provides relevant information on the star formation and chemical histories of the host galaxies. Despite the multiple observational as well as theoretical projects undertaken in the last few years, our currently existing knowledge of both the stellar formation processes and chemical evolution of galaxies is, in general, incomplete. Even for the galaxies in the Local Group, our present understanding is definitely limited. In this state of affairs, the stellar cluster systems of the Magellanic Clouds, on account of their proximity, richness, and variety, may furnish us with the ideal ground to conduct a detailed examination of the processes mentioned above.

Detailed colour-magnitude diagrams (CMDs) of star clusters can hardly be obtained beyond the Local Group. At the distance of M 31 and M 33, HST CMDs reach little below the horizontal branch (e.g., Holland et al. 1997; Sarajedini et al. 2000). Integrated light methods are the main tools to study star

Send offprint requests to: A. V. Ahumada,

e-mail: andrea@mail .oac.uncor.edu

* Based on observations made at Complejo Astronómico El Leoncito, which is operated under agreement between the Consejo Nacional de Investigaciones Científicas y Técnicas de la República Argentina and the National Universities of La Plata, Córdoba and San Juan, Argentina. clusters in more distant galaxies, such as colours (e.g., star clusters in the Fornax giant galaxy NGC 1399, Ostrov et al. 1998) and spectra (e.g., in the Virgo giant galaxy NGC 4472, Beasley et al. 2000). Efforts to create reference spectra of star clusters and grids of their properties to be used as templates for different ages and metallicities in the study of composite stellar populations were made by Bica \& Alloin (1986a,b, hereafter BA86a, BA86b), Bica (1988), and Piatti et al. (2002). Star cluster templates are also useful to derive parameters of unstudied clusters, e.g. Galactic open clusters (Santos \& Bica 1993; Piatti et al. 1999; Ahumada et al. 2000, 2001).

The sample of reference clusters in BA86a and BA86b was dedicated mostly to Galactic and Large Magellanic Cloud (LMC) clusters; only four Small Magellanic Cloud (SMC) objects were included therein. The goal of the present study is to collect and analyze a large sample of SMC clusters in view of: (i) studying the integrated light properties of such metal deficient clusters, especially the young and intermediate-age ones; and (ii) making them available as template spectra for studies of star clusters in more distant dwarf galaxies.

\section{Cluster sample and observations}

The determination of SMC cluster parameters, particularly age, is fundamental to understand the structure and evolution of 
Table 1. Cluster sample coordinates.

\begin{tabular}{lcccc}
\hline \hline Cluster & $\begin{array}{c}l \\
\left.{ }^{\circ}\right)\end{array}$ & $\begin{array}{c}b \\
\left({ }^{\circ}\right)\end{array}$ & $\begin{array}{c}\alpha_{2000} \\
(\mathrm{~h} \mathrm{~m} \mathrm{~s})\end{array}$ & $\begin{array}{c}\delta_{2000} \\
\left({ }^{\circ} \prime \prime\right)\end{array}$ \\
\hline NGC 796, L 115, WG 9, ESO30SC6 & 297.0142 & -42.1502 & 015645 & -741310 \\
L 114, WG 4, ESO30SC5 & 297.6283 & -42.1604 & 015019 & -742123 \\
NGC 643, L 111, ESO29SC50 & 299.3374 & -41.2661 & 013500 & -753324 \\
HW 73 & 300.0735 & -45.6632 & 011626 & -711935 \\
NGC 416, K 59, L 83, ESO29SC32 & 301.1696 & -44.7155 & 010759 & -722123 \\
IC 1624, K 52, L 76, ESO51SC17, SMC_OGLE 149 & 301.4132 & -45.0441 & 010522 & -720236 \\
L 56, SMC-S26, SMC_OGLE 109 & 302.2789 & -44.8561 & 005731 & -721547 \\
NGC 306, K 33, L 50, ESO29SC23 & 302.6292 & -44.8852 & 005415 & -721429 \\
L 48, ESO51SC6 & 302.7001 & -45.7287 & 005328 & -712356 \\
NGC 290, L 42, ESO29SC19, SMC_OGLE69 & 302.9524 & -43.9669 & 005114 & -730941 \\
NGC 265, K 24, L 34, ESO29SC14, SMC_OGLE 39 & 303.3484 & -43.6504 & 004712 & -732840 \\
NGC 256, K 23, L 30, ESO29SC11, SMC_OGLE 32 & 303.4747 & -43.6158 & 004554 & -733026 \\
NGC 241, K 22w, L 29w, ESO29SC6w, SMC_OGLE 17 & 303.7088 & -43.6761 & 004333 & -732629 \\
NGC 242, K 22e, L 29e, SMC_OGLE 18 & 303.7006 & -43.6758 & 004334 & -732644 \\
L 28 & 303.8188 & -44.5251 & 004259 & -723520 \\
NGC 121, K 2, L 10, ESO50SC12 & 305.7105 & -45.4583 & 002647 & -713212 \\
\hline
\end{tabular}

Cluster identifications are from Lindsay (1958, L), Kron (1956, K), Lauberts (1982, ESO), Westerlund \& Glaspey (1971, WG). Hodge \& Wright (1974, HW) and Pietrzyński \& Udalski (1999, OGLE).

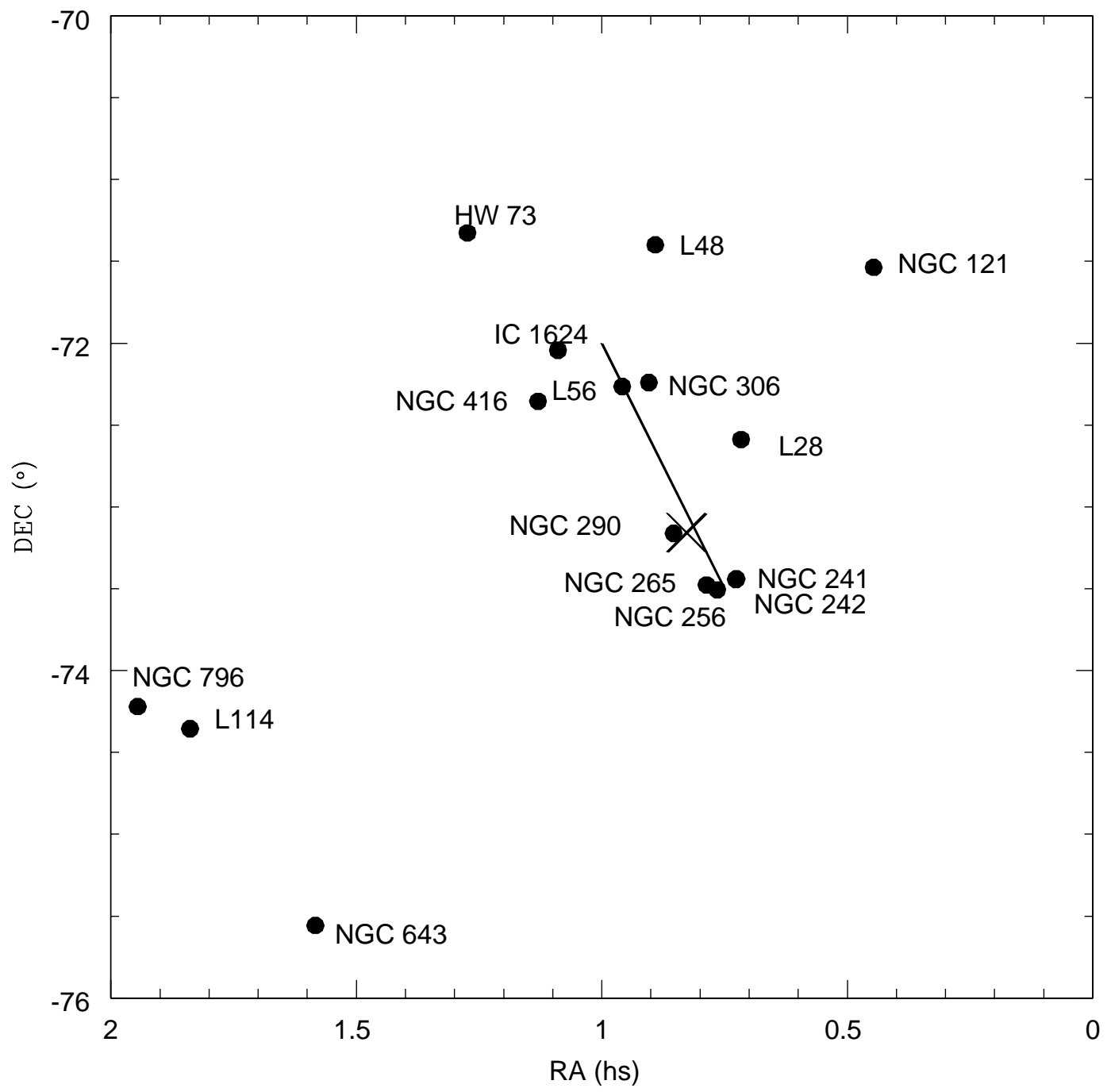

Fig. 1. The position of the 16 studied star clusters (filled circles) with relation to the SMC bar (straight line) and optical centre (cross). 
Table 2. Journal of observations.

\begin{tabular}{lcccc}
\hline \hline Cluster & $\begin{array}{c}\text { Date } \\
(\mathrm{UT})\end{array}$ & $\begin{array}{c}\text { Exposure } \\
\text { Time }(\mathrm{s})\end{array}$ & $\begin{array}{c}\text { FWHM } \\
\left({ }^{\prime \prime}\right)\end{array}$ & $\begin{array}{c}\text { Area } \\
\left("{ }^{\prime \prime}\right)\end{array}$ \\
\hline NGC 796 & $10 / 11 / 99$ & $5 \times 900$ & 2.3 & $66 \times 72$ \\
& $10 / 25 / 00$ & $2 \times 600$ & 2.8 & \\
L 114 & $10 / 21 / 98$ & $2 \times 900$ & 2.3 & $84 \times 70$ \\
& $10 / 11 / 99$ & $2 \times 900$ & 2.3 & \\
NGC 643 & $10 / 26 / 98$ & $3 \times 900$ & 3.1 & $130 \times 130$ \\
& $10 / 11 / 99$ & $4 \times 900$ & 2.3 & \\
HW 73 & $10 / 26 / 98$ & $3 \times 900$ & 3.1 & $60 \times 54$ \\
& $10 / 11 / 99$ & $2 \times 900$ & 2.3 & \\
NGC 416 & $10 / 25 / 00$ & $2 \times 600$ & 3.0 & $50 \times 50$ \\
IC 1624 & $10 / 12 / 99$ & $4 \times 900$ & 2.8 & $54 \times 54$ \\
L 56 & $10 / 13 / 99$ & $4 \times 900$ & 2.8 & $57 \times 57$ \\
NGC 306 & $10 / 21 / 98$ & $2 \times 900$ & 2.3 & $65 \times 65$ \\
& $10 / 13 / 99$ & $2 \times 900$ & 2.8 & \\
L 48 & $10 / 22 / 98$ & $2 \times 900$ & 2.8 & $57 \times 57$ \\
& $10 / 13 / 99$ & $2 \times 900$ & 2.8 & \\
NGC 290 & $10 / 13 / 99$ & $4 \times 900$ & 2.8 & $65 \times 65$ \\
NGC 265 & $10 / 13 / 99$ & $4 \times 900$ & 2.8 & $70 \times 70$ \\
NGC 256 & $10 / 12 / 99$ & $4 \times 900$ & 2.8 & $54 \times 54$ \\
NGC 241 & $10 / 25 / 00$ & $3 \times 600$ & 3.0 & $57 \times 57$ \\
NGC 242 & $10 / 25 / 00$ & $3 \times 600$ & 3.0 & $45 \times 57$ \\
L 28 & $10 / 21 / 98$ & $3 \times 900$ & 2.3 & $60 \times 60$ \\
& $10 / 11 / 99$ & $3 \times 900$ & 2.3 & \\
NGC 121 & $10 / 11 / 99$ & $3 \times 900$ & 2.3 & $228 \times 180$ \\
\hline
\end{tabular}

this galaxy. Concentrated clusters, with small angular diameter are certainly the most suitable to carry out integrated spectroscopy observations. This is so because the cluster as well as the surrounding background regions must extend along the slit. Besides, the angular diameter requirement results from the fact that the cluster integrated spectrum must reflect the synthesis of its stellar content.

In this study we have selected 16 relatively populous and compact SMC clusters to allow good star sampling in the integrated spectra. One of the selected clusters, the well-known intermediate-age SMC cluster NGC 416 (Mighell et al. 1998), was included in the sample for comparison purposes. The observed star cluster sample is given in Table 1, where their designations in different catalogues are provided. Figure 1 shows their positions in relation to the SMC bar. Ten clusters (NGC 241, NGC 242, NGC 256, NGC 265, NGC 290, L 28, L 56, NGC 306, NGC 416 and IC 1624) are projected on the SMC main body. If the position $(\mathrm{J} 2000): 00^{\mathrm{h}} 49^{\mathrm{m}} 27^{\mathrm{s}}$, $-73^{\circ} 09^{\prime} 30^{\prime \prime}$ is assumed to be the centre of the SMC bar, L 48 is located at $\approx 1.7^{\circ}$ to the north, NGC 121 about the same amount to the northwest and HW $73 \approx 2.5^{\circ}$ to the northeast. NGC 643 at $\approx 3.5^{\circ}$ to the southeast is among the outermost SMC clusters. Finally, L 114 and NGC 796 at $\approx 3.4^{\circ}$ southeast of the bar, are projected on the Bridge linking the SMC and LMC.

The observations were carried out with the $2.15 \mathrm{~m}$ telescope at the Complejo Astronómico El Leoncito (CASLEO, San Juan, Argentina) in three runs in October 1998, 1999 and 2000, respectively. In all these runs we employed a CCD camera containing a Tektronics chip of $1024 \times 1024$ pixels attached to a REOSC spectrograph (simple mode), the size of each pixel being $24 \mu \mathrm{m} \times 24 \mu \mathrm{m}$. The slit was oriented in the east-west direction and the observations were performed scanning the slit across the objects in the north-south direction in order to get a proper sampling of cluster stars. The long slit, corresponding to $4.7^{\prime}$ on the sky, allowed us to sample regions of the background sky. A grating of 300 grooves/mm was used. The spectral coverage was (3600-6800) $\AA$, with an average dispersion in the observed region of $\approx 140 \AA / \mathrm{mm}(3.46 \AA / \mathrm{pix})$. The slit width was 4 "' 2 , resulting in a resolution of $\approx 14 \AA$, as measured by the mean full width half maximum $(F W H M)$ of the comparison lines. At least two exposures of 10 min of each object were taken in order to correct for cosmic rays. Standard stars (Stone \& Baldwin 1983) were also observed for flux calibrations, and $\mathrm{He}-\mathrm{Ne}-\mathrm{Cu}$ lamp exposures were taken for wavelength calibrations. Bias, dome and twilight sky flat fields were taken and employed in the reductions.

The journal of observations is provided in Table 2, by columns: (1) cluster designation, (2) date of observation, (3) number of exposures and duration in seconds, (4) FWHM in arcseconds, determined by the seeing during observation, and (5) total area of the cluster scanned in our observations.

The reductions were carried out with the IRAF software package following standard procedures at the Observatorio Astronómico de la Universidad Nacional de Córdoba. In Fig. 2 we present the flux-calibrated integrated spectra of the observed clusters in relative flux units, normalized to $F_{\lambda}=1$ at $\lambda=5800 \AA$. The spectra are shifted by arbitrary constants for comparison purposes. To improve the signal-to-noise ratio, the spectrum of NGC 121 was averaged with that previously obtained by BA86a. The spectral lines and different slopes of 


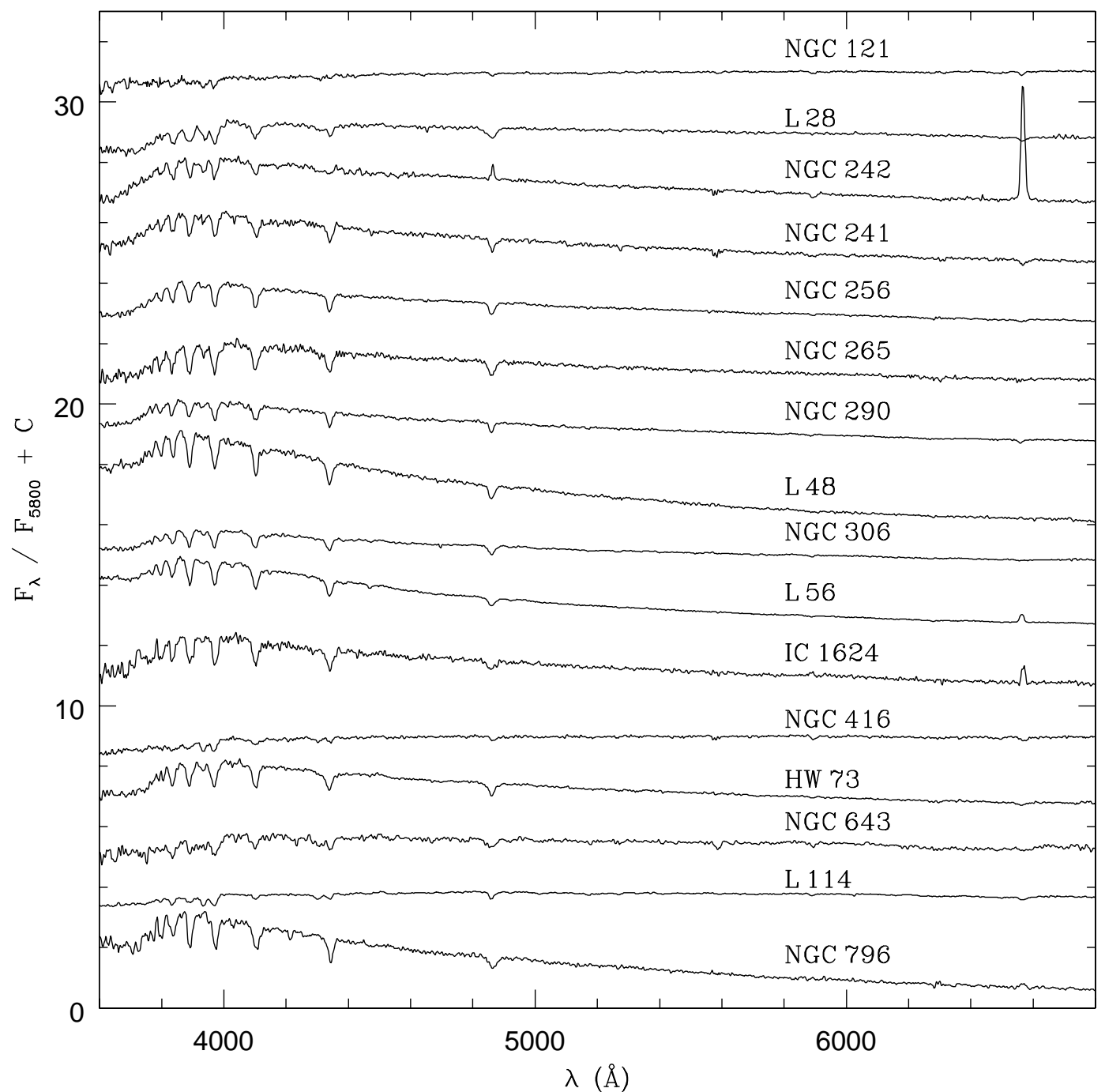

Fig. 2. Observed integrated spectra of 16 SMC clusters. Spectra are in relative $F_{\lambda}$ units normalised at $\lambda=5800 \AA$ A. Constants have been added to the spectra for clarity, except for the bottom one.

Table 3. Measurements of equivalent widths of four Balmer lines.

\begin{tabular}{lcccc}
\hline \hline & $\mathrm{H}_{\delta}$ & $\mathrm{H}_{\gamma}$ & $\mathrm{H}_{\beta}$ & $\mathrm{H}_{\alpha}$ \\
Windows & $(4082-4124) \AA$ & $(4318-4364) \AA$ & $(4846-4884) \AA$ & $(6540-6586) \AA$ \\
Cluster & & & & \\
\hline NGC 796 & $7.57 \pm 0.09$ & $7.77 \pm 0.07$ & $6.40 \pm 0.17$ & $-3.89 \pm 0.08$ \\
L 114 & $5.68 \pm 0.08$ & $5.26 \pm 0.10$ & $4.71 \pm 0.05$ & $3.78 \pm 0.06$ \\
NGC 643 & $8.56 \pm 0.17$ & $7.19 \pm 0.20$ & $6.40 \pm 0.06$ & $3.86 \pm 0.10$ \\
HW 73 & $8.58 \pm 0.10$ & $8.51 \pm 0.10$ & $6.19 \pm 0.07$ & $3.90 \pm 0.11$ \\
NGC 416 & $5.30 \pm 0.01$ & $2.80 \pm 0.01$ & $2.47 \pm 0.06$ & $2.64 \pm 0.08$ \\
IC 1624 & $7.65 \pm 0.04$ & $8.88 \pm 0.07$ & $5.00 \pm 0.09$ & $-8.77 \pm 0.08$ \\
L 56 & $6.10 \pm 0.06$ & $6.33 \pm 0.05$ & $3.81 \pm 0.05$ & $-5.10 \pm 0.08$ \\
NGC 306 & $6.49 \pm 0.08$ & $5.89 \pm 0.04$ & $5.09 \pm 0.08$ & $1.90 \pm 0.05$ \\
L 48 & $6.05 \pm 0.04$ & $7.03 \pm 0.05$ & $4.74 \pm 0.02$ & $1.23 \pm 0.07$ \\
NGC 290 & $6.30 \pm 0.11$ & $4.67 \pm 0.01$ & $4.63 \pm 0.05$ & $2.54 \pm 0.02$ \\
NGC 265 & $10.00 \pm 0.09$ & $9.48 \pm 0.11$ & $8.29 \pm 0.11$ & $3.38 \pm 0.04$ \\
NGC 256 & $7.87 \pm 0.07$ & $7.52 \pm 0.05$ & $5.97 \pm 0.05$ & $3.32 \pm 0.05$ \\
NGC 241 & $7.02 \pm 0.02$ & $5.40 \pm 0.02$ & $5.90 \pm 0.04$ & $5.20 \pm 0.02$ \\
NGC 242 & $4.31 \pm 0.01$ & $2.22 \pm 0.01$ & $-2.5 \pm 0.1$ & $-78.7 \pm 0.1$ \\
L 28 & $10.18 \pm 0.07$ & $8.20 \pm 0.17$ & $6.88 \pm 0.21$ & $3.71 \pm 0.06$ \\
NGC 121 & $3.30 \pm 0.03$ & $3.78 \pm 0.05$ & $1.57 \pm 0.03$ & $2.08 \pm 0.01$ \\
\hline
\end{tabular}




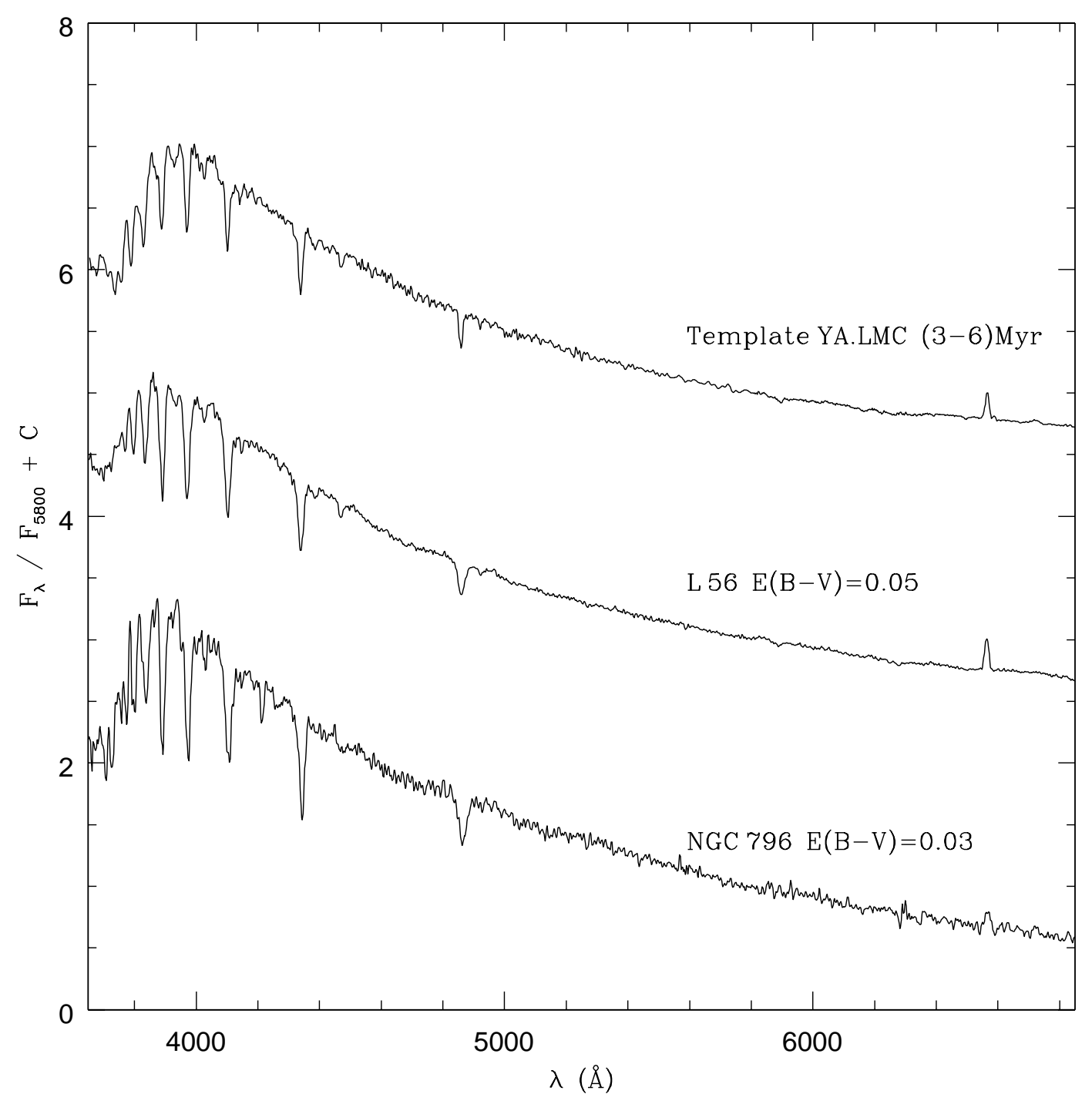

Fig. 3. Comparison of reddening-corrected spectra of NGC 796 (bottom) and L 56(middle) with the template which best matches them (top). Units as in Fig. 2.

the continuum energy distributions in Fig. 2 are primarily the result of age effects. We also explore the low metallicity effects and low reddening implications.

\section{Determination of cluster fundamental parameters}

The equivalent widths (EWs) of the Balmer lines were measured with the spectral analysis program SPEED (Schmidt 1988) taking into account both the spectral windows and flux points as defined by BA86a. Two alternative fits using high and low continuum tracings were made in order to take into account the spectral noise in the measurements of the Balmer-line EWs. The resulting uncertainties in the EWs are found to be smaller than those associated with the intrinsic dispersions in Table 2 of BA86b. The results of the measurements are presented in Table 3, where the EWs are given in Angström units $(\AA)$. Bica (1988), Bica et al. (1990), Santos et al. (1995) and Piatti et al. (2002) created sets of template cluster spectra with known parameters, grouped according to their evolutionary stages.
In this study we have simultaneously estimated age and foreground reddening values by comparing the continuum and lines of the observed cluster spectra with those of template cluster spectra. A direct reddening-independent age estimate was first obtained from EWs of the Balmer absorption lines in each spectrum by interpolating these values in the calibration of BA86b. In the present study, we adopted $t=12 \mathrm{Gyr}$ for globular cluster ages. Then, we selected an appropriate set of template spectra according to the age provided by the Balmer lines and varied reddening and template to get the best match of continuum, Balmer and metal lines of the observed spectrum to that of the template that most resembles it. The reddening corrections were performed employing the interstellar absorption law derived by Seaton (1979) and adopting $R=A_{v} / E(B-V)=3.0$. Table 4 shows the age and foreground reddening values obtained for the cluster sample. It is worth noticing that the uncertainty in the adopted reddening constitutes the minimum reddening variation necessary to differentiate the cluster spectrum from that of the matching template. As Table 4 also makes clear, the uncertainties in the $E(B-V)$ 


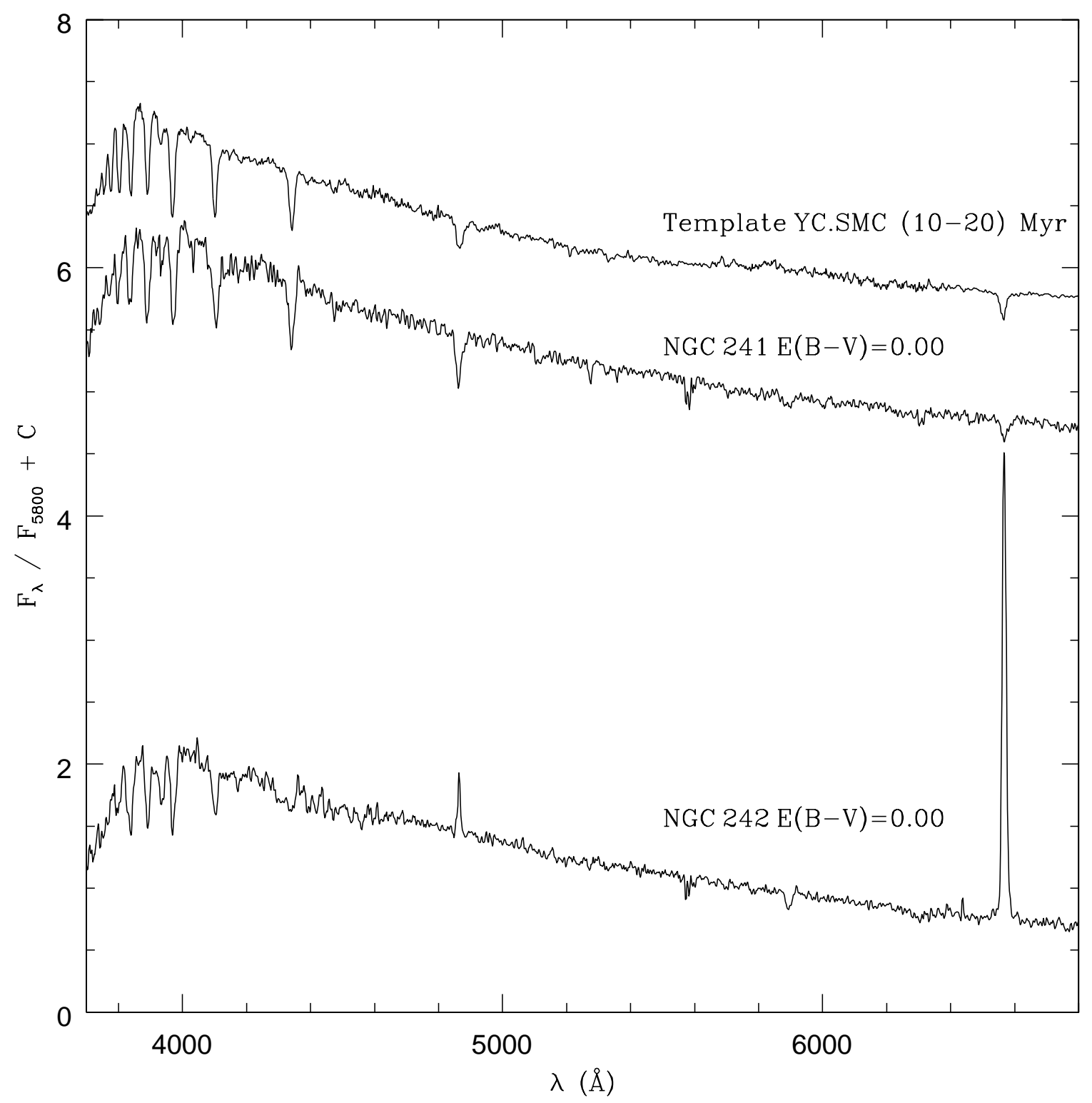

Fig. 4. Observed integrated spectra of NGC 242 (bottom) and NGC 241 (middle) and the template which best matches the spectrum of NGC 241 and the continuum of NGC 242 (top). Units as in Fig. 2.

determinations vary from 0.01 to 0.03 magnitudes. Were the age assignment to be off by one template class, the uncertainty mentioned above would be only slightly greater.

The following templates from Bica et al. (1990) in the spectral range (5600-10000) $\AA$ were found to be useful in this study: YC.LMC (12-35 Myr), YD.LMC (35-50 Myr), YE.LMC (50-85 Myr), YF.LMC (85-130 Myr) and YG.LMC (130-250 Myr). We have also used the following templates from Santos et al. (1995) in the spectral region (3600-5900) $\AA$ : YA.LMC (3-6 Myr), YDE.LMC (35-65 Myr) and YC.SMC (10-20 Myr), as well as the Y4 (500 Myr), G4 (globular cluster age and $\left.\left[Z / Z_{\odot}\right]=-1.5\right)$ and G5 (globular cluster age and $\left.\left[Z / Z_{\odot}\right]=-1.9\right)$ templates in the range (3500-9700) $\AA$ from Bica (1988). The spectral regions corresponding to $\lambda>5900 \AA$ of the Santos et al. (1995) templates, were taken from Bica et al. (1990). We have also used the Int1 (LMC IACs around 1 Gyr) template in the range (3600-6600) $\AA$ from Dutra et al. (1998).

\section{Discussion}

The reddening-corrected spectra of the two youngest clusters of the present sample (L 56 and NGC 796) are compared with the YA.LMC (3-6 Myr) template in Fig. 3, while Fig. 4 shows a comparison of the reddening-corrected spectra of the cluster pair NGC 241/NGC 242 with the YC.SMC (10-20 Myr) template, which best matches them. Figures 5 and 6 show comparisons of the reddening-corrected spectra of four and two SMC clusters with the YDE.LMC (35-65 Myr) and YC.LMC (12-35 Myr) templates, respectively, which best match them. The integrated spectrum of the well known SMC cluster NGC 121 is compared with that of the G4 (12 Gyr) template in Fig. 7. Finally, the three clusters found to be of intermediateage (see below) are compared with the templates Y4 (500 Myr), Int1 (1 Gyr), G5 (12 Gyr) and NGC 416 (5.6 Gyr, Mighell et al. 1998) in Figs. 8 and 9. As can be seen in Figs. 3 to 9, the match 


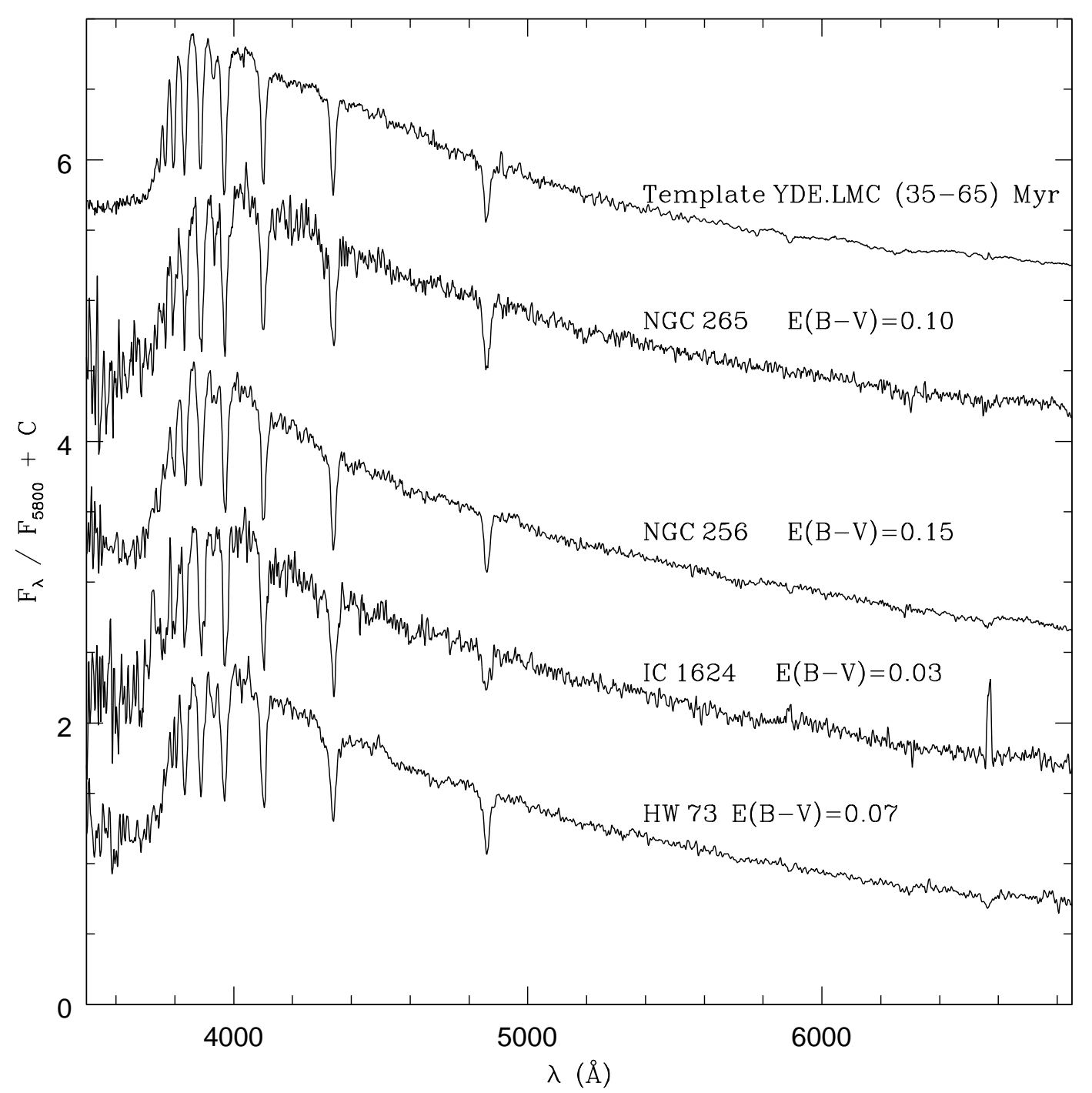

Fig. 5. Comparison of reddening-corrected spectra of HW 73 (bottom), IC 1624, NGC 256 and NGC 265 and the template which best matches the spectra (top). Units as in Fig. 2.

of all the new cluster spectra with those of the selected templates is really excellent.

About half of the clusters here studied have not been previously observed. Using CMDs built from the OGLE database and Padova isochrones, Pietrzynski \& Udalski (1999) obtained $158 \mathrm{Myr}$ for IC 1624, $100 \mathrm{Myr}$ for NGC 256 and NGC 265, 79 Myr for NGC 241 and NGC 242, $50 \mathrm{Myr}$ for L 56 and 40 Myr for NGC 290. Cassatella et al. (1996) obtained 23 Myr for L 56 from the ultraviolet colour index C(15$31)$. This value is in excellent agreement with the one here derived from the Balmer lines. Also, using the OGLE database and Padova isochrones, de Oliveira et al. (2000) studied the cluster pair NGC 241 and NGC 242, obtaining 55 and $65 \mathrm{Myr}$, respectively. The isophotal contours obtained by de Oliveira et al. (2000) clearly show a bridge linking them, which could be interpreted as a sign of interaction. Basically, for such blue star clusters, age determinations above and the present ones agree within a factor 2-3 (see Table 4). Differences can be explained by crowding in the cluster area, background and cluster contamination by bright stars, and age calibrations based on classical or overshoot models. In particular, the age calibration used by BA86b on which we rely here, is based on classical models. Using integrated $U B V$ observations, van den Bergh (1981) classified NGC 256, NGC 265 and NGC 306 as young clusters, in good agreement with the present age determinations. However, he also reports L 114 as a young cluster, which is not supported by the present observations. Pietrzynski \& Udalski (1999) also determined $E(B-V)$ colour excesses from CMDs for the SMC clusters IC 1624, L 56, NGC 290, NGC 265, NGC 256, NGC 241 and NGC 242. The values they found show good agreement with those here derived, the mean difference between their $E(B-V)$ values and the present ones being $\Delta E(B-V)=0.03 \pm 0.05$.

The spectra of L 48 and NGC 796 are the bluest ones in the sample (Fig. 2). In particular, that of L48 is bluer than the template corresponding to its Balmer age (Table 4). This cluster is a rare case of a young object outside the SMC main body. NGC 796 is projected on the Bridge linking the SMC and LMC, while L 48 is far north in the SMC (Fig. 1). Note that the employed templates are composed mostly of clusters 


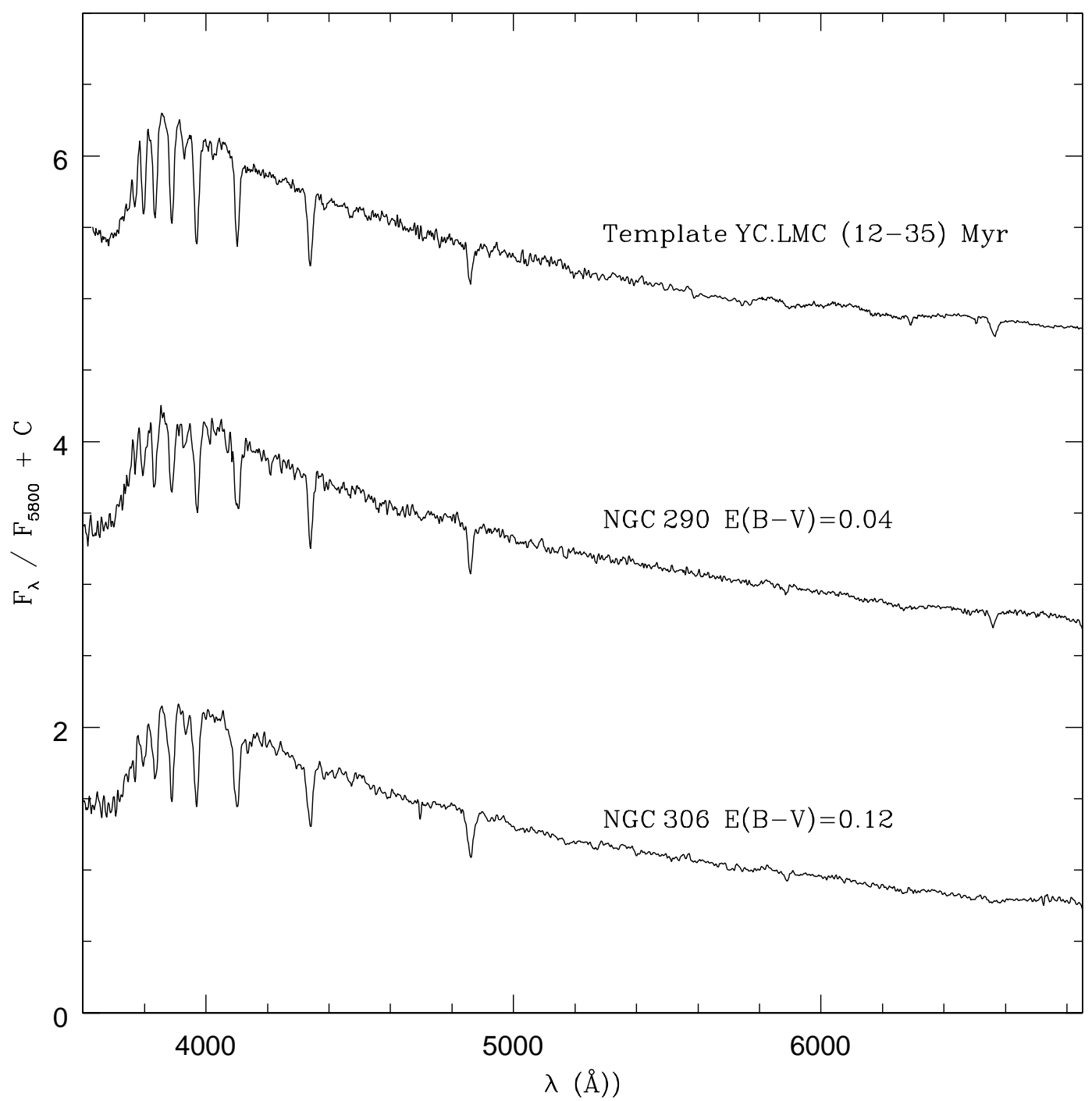

Fig. 6. Comparison of reddening-corrected spectra of the SMC clusters NGC 306 and NGC 290 with the template which best matches them (top). Units as in Fig. 2.

Table 4. Reddening and age determinations.

\begin{tabular}{lcccccc}
\hline \hline Cluster & $E(B-V)$ & $\begin{array}{c}\text { CMD Age } \\
(\mathrm{Myr})\end{array}$ & $\begin{array}{c}\text { Balmer Age } \\
(\mathrm{Myr})\end{array}$ & Adopted Template & $\begin{array}{c}\text { Template Age } \\
(\mathrm{Myr})\end{array}$ & $\begin{array}{c}\text { Adopted age } \\
(\mathrm{Myr})\end{array}$ \\
\hline NGC 796 & $0.03 \pm 0.03$ & - & 50 & YA.LMC & $3-6$ & $6 \pm 10$ \\
L 114 & $0.00 \pm 0.02$ & - & 6000 & NGC 416 & 5600 & $(5600 \pm 500)$ \\
NGC 643 & $0.08 \pm 0.03$ & - & 2000 & Int1 & 1000 & $(1000 \pm 200)$ \\
HW 73 & $0.07 \pm 0.01$ & - & 60 & YDE.LMC & $35-65$ & $50 \pm 10$ \\
NGC 416 & $0.00 \pm 0.01$ & 5600 & 4000 & - & 5600 & $5600 \pm 500$ \\
L 56 & $0.05 \pm 0.02$ & 50 & 23 & YA.LMC & $3-6$ & $6 \pm 10$ \\
IC 1624 & $0.03 \pm 0.02$ & 158 & 40 & YDE.LMC & $35-65$ & $50 \pm 10$ \\
NGC 306 & $0.12 \pm 0.01$ & - & 30 & YC.LMC & $12-35$ & $30 \pm 10$ \\
L 48 & - & - & 40 & - & - & $40 \pm 10$ \\
NGC 290 & $0.04 \pm 0.02$ & 40 & 40 & YC.LMC & $12-35$ & $30 \pm 10$ \\
NGC 265 & $0.10 \pm 0.01$ & 100 & 70 & YDE.LMC & $35-65$ & $50 \pm 10$ \\
NGC 256 & $0.15 \pm 0.02$ & 100 & 50 & YDE.LMC & $35-65$ & $50 \pm 10$ \\
NGC 241 & $0.00 \pm 0.02$ & 55 & 30 & YC.SMC & $10-20$ & $20 \pm 10$ \\
NGC 242 & $0.00 \pm 0.02$ & 65 & - & YC.SMC & $10-20$ & $20 \pm 10$ \\
L 28 & $0.03 \pm 0.02$ & - & 1200 & Int1 & 1000 & $(1000 \pm 200)$ \\
NGC 121 & $0.02 \pm 0.07$ & 10000 & 12000 & G4 & 12000 & $12000 \pm 1000$ \\
\hline
\end{tabular}




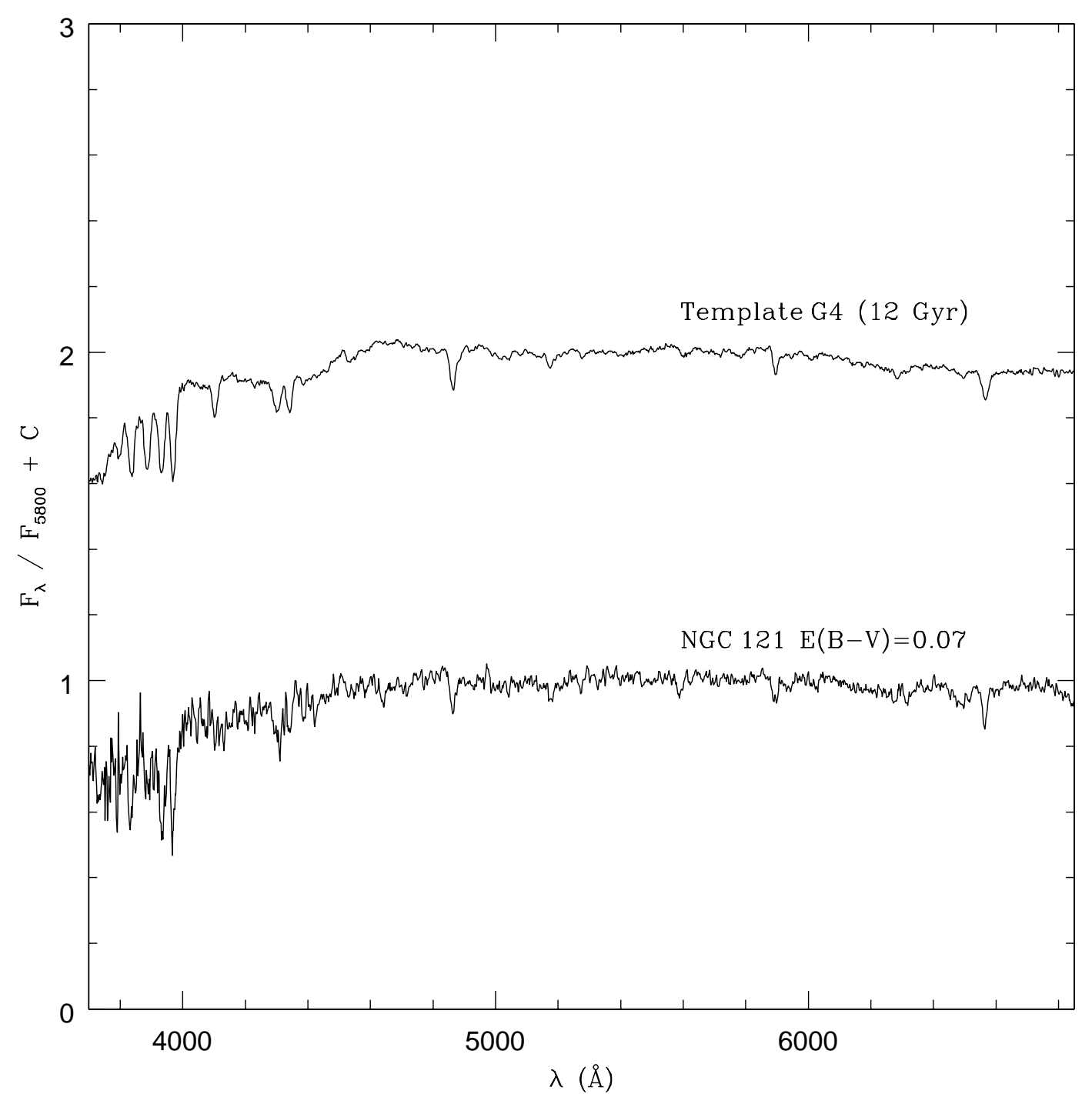

Fig. 7. The integrated spectrum of NGC 121 corrected for the derived reddening $E(B-V)=0.07$ (bottom) and the template which best matches the spectrum (top). Units as in Fig. 2.

in the main body of the LMC, and are not corrected for internal reddening. A possible explanation is that $\mathrm{L} 48$ has atypically low internal reddening for its age, by the fact that it is not a main body cluster within a denser interstellar medium. Note also that several clusters in the range 20-50 Myr have $\mathrm{H}_{\alpha}$ in emission or filling the absorption (Fig. 2). Such effect is not due to nebular emission, since it is not accompanied by forbidden lines. They arise from circumstellar envelopes, like in Be stars (Bica et al. 1990). In particular, NGC 242 (Fig. 2) appears to be a prominent case, stochastically rich with emission line stars as compared to its twin NGC 241.

The integrated spectra of L 28, NGC 643 and L 114 show typical features of intermediate-age clusters (IACs). Note the stronger metal lines, in particular $\mathrm{K} \mathrm{CaII}$, which indicates that the clusters are older than the Y4 template from Bica (1988). Contrary to what happens with the younger star clusters, wherein the upper main sequence dominates their visible spectra, the IACs are known to share similar spectral properties with metal-poor globular clusters, especially in the blueviolet region (e.g., BA86a, BA86b). Since the SMC is metal deficient, this similarity is further enhanced because of the weaker metal lines and overall blanketing. This is the case of L 114, whose spectral features are comparable to those in the G5 template (Fig. 8) but its Balmer lines indicate a much younger age. As shown in Fig. 8, the integrated spectrum of L 114 exhibits a remarkable similarity to that of the $5.6 \mathrm{Gyr}$ old SMC cluster NGC 416 (Mighell et al. 1998). L 114, projected towards the Bridge, is very probably an IAC and a CMD will be important for a more accurate age determination. On the other hand, although the observed spectra of L 28 and NGC 643 compare reasonably well with the Y4 template (Fig. 9), both clusters are very probably older than $500 \mathrm{Myr}$, as suggested by the Balmer and metal lines, especially K CaII. Figure 9 shows the spectra corrected for the derived interstellar reddenings $E(B-V)=0.03$ and 0.08 , respectively, and the Int1 (LMCIACs around $1 \mathrm{Gyr}$ ) template which best matches the cluster spectra. For SMC IACs, reddening and blanketing effects compete, and one can hardly compare their integrated spectra with those of LMC and Galactic templates. This is why we derive here only tentative ages (in parentheses in Table 4) 


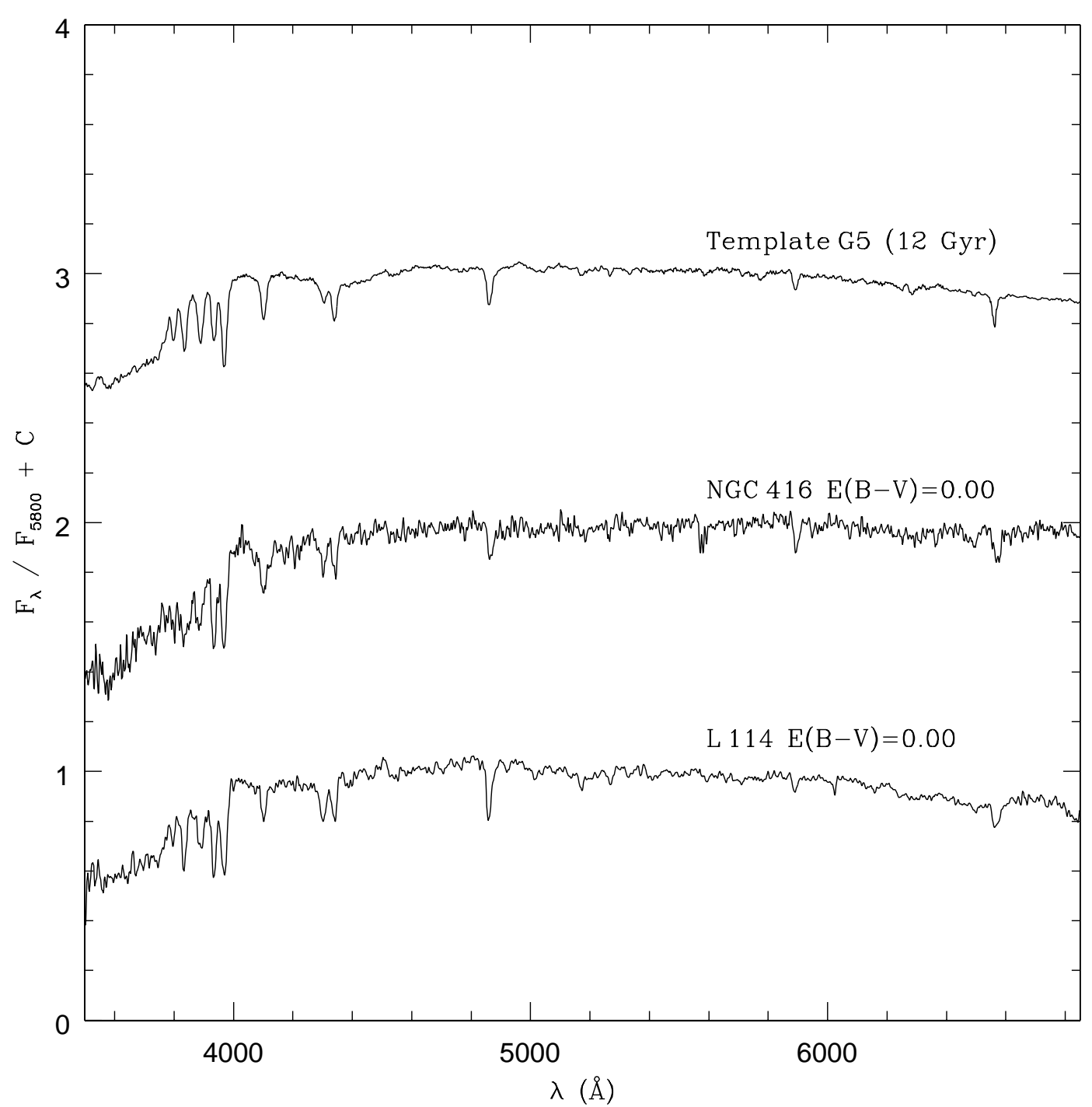

Fig. 8. Comparison of the observed integrated spectra of L 114 and NGC 416 with the G5 (globular cluster age and $\left.\left[Z / Z_{\odot}\right]=-1.9\right)$ template. Units as in Fig. 2.

for these three IACs, based on the Balmer lines method. The age derived for NGC 643 is in good agreement with the value found by Bica et al. (1986c, hereafter BDP86) using $\mathrm{H}_{\beta}$ and G-band photometry.

NGC 121 has been studied by many groups and the results found here are in very good agreement with those. For example, BDP86 found an age older than $10 \mathrm{Gyr}$ and a metallicity: $\left[Z / Z_{\odot}\right]=-1.3$, while in a recent work Shara et al. (1990) obtained an age of $10 \pm 2 \mathrm{Gyr}$ and $\left[Z / Z_{\odot}\right]=-1.4$, both of which are similar to the ones here reported.

Due to the remarkable differences in age existing among the clusters studied in this work, their metallicities are quite likely to differ just as much. For the younger clusters in this sample, it would be reasonable to adopt the present-day SMC abundance of $[\mathrm{Fe} / \mathrm{H}] \approx-0.6 \mathrm{dex}$ (Da Costa \& Hatzidimitriou $1998)$, while for those of intermediate-age, $[\mathrm{Fe} / \mathrm{H}]$ values ranging from approximately -0.9 to -1.6 should be expected (see, e.g., Olszewski et al. 1996; Rich et al. 2000; Piatti et al. 2001). Since the spectrum of young star clusters is dominated by hot stars, the metallicity of the templates is not crucial for the comparisons. For intermediate ages around 5 Gyr, we have a template cluster in the SMC itself, NGC 416. Age uncertainties arising from metallicities are probably more important for clusters around 1-2 Gyr, since blanketing effects affect the blue violet region considerably. In the latter case, age uncertainties of around 1 Gyr may be expected.

Finally, we point out that the age resolution and range of the present SMC objects, form a significant data set to be used as templates for other studies of star clusters in galaxies, especially dwarf ones.

\section{Conclusions}

We have studied a sample of 16 SMC concentrated star clusters by means of integrated spectroscopy in the range (3600-6800) $\AA$. We have estimated ages and foreground reddening values from the comparison of the cluster integrated spectra with template cluster spectra. Eleven clusters (HW 73, IC 1624, L 48, L 56, NGC 241, NGC 242, NGC 256, NGC 265, NGC 290, NGC 306 and NGC 796) are young with 


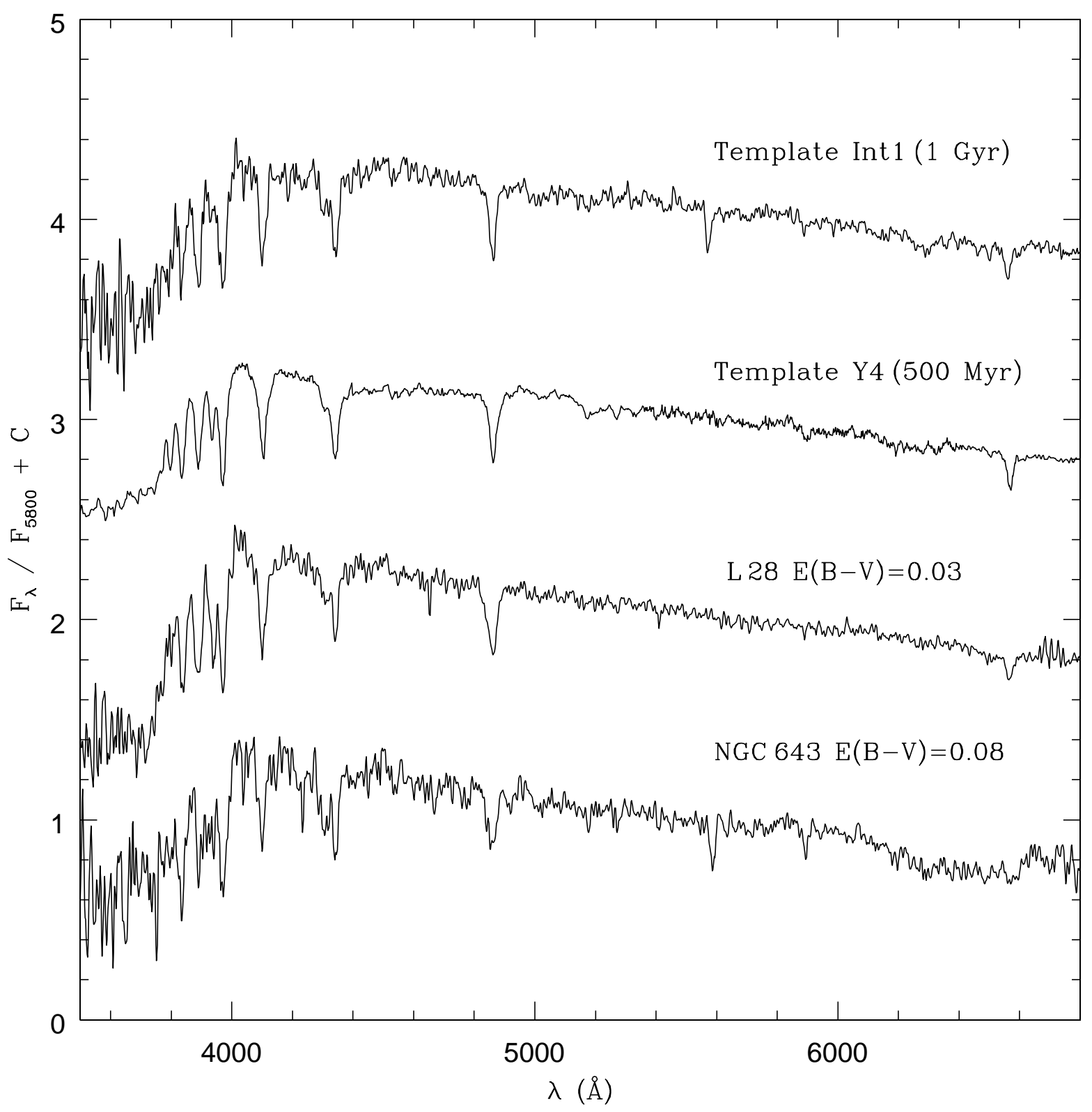

Fig. 9. Comparison of the reddening-corrected spectra of NGC 643 and L 28 with the 500 Myr and 1 Gyr templates. Units as in Fig. 2.

ages ranging between 6 and $50 \mathrm{Myr}$, three (L 114, L 28 and NGC 643) were found to be of intermediate age (1-6 Gyr), the remaining two objects being the well known old and intermediate-age clusters NGC 121 and NGC 416, respectively. Only tentative ages were estimated for the three IACs. The derived reddening values range from $E(B-V)=0.00$ in four clusters to $E(B-V)=0.15$ in NGC 256, although no $E(B-V)$ colour excess was derived for the bluest cluster L 48 . The spectrum of L 48 is bluer than the template corresponding to its Balmer age, probably due to the fact that this cluster suffers from atypically low internal reddening. It is remarkable that three young clusters, namely L 48, HW 73 and NGC 796, are outlying SMC clusters, at projected distances comparable to those of several well-known intermediate-age outlyers and that of the old cluster NGC 121.

We conclude that much care is necessary in the interpretation of integrated spectra of IACs in dwarf galaxies.
The present work is a step forward in their interpretation. In a forthcoming paper, we will determine ages for some of these clusters from CMDs, and derive templates in this specific age and metallicity range for integrated studies in distant galaxies, especially dwarf ones.

Acknowledgements. We are gratefully indebted to the CASLEO staff for their hospitality and support during the two observing runs. The authors acknowledge use of the CCD and data acquisition system supported under U.S. National Science Foundation grant AST-90-15827 to R. M. Rich. Thanks are also due to J. F. C. Santos Jr. who kindly sent us the near-infrared portions of several Magellanic Cloud templates. We also thank the referee U. Fritze von Alvensleben for her valuable comments and suggestions. Dutra acknowledges FAPESP for a post-doc fellowship (proc. 00/11864-6). This work was partially supported by the Argentinian institutions CONICET, SECYT (Universidad Nacional de Córdoba), AGENCIA CÓRDOBA CIENCIA, and AGENCIA NACIONAL DE 
PROMOCIÓN CIENTÍFICA Y TECNOLÓGICA (ANPCYT), and the Brazilian institutions CNPq and FINEP.

\section{References}

Ahumada, A. V., Clariá, J. J., Bica, E., \& Piatti, A. E. 2000, A\&AS, 141,79

Ahumada, A. V., Clariá, J. J., Bica, E., Dutra, C. M., \& Torres, M. C. 2001, A\&A, 377, 845

Beasley, M. A., Sharples, R. M., Bridges, T. J., et al. 2000, MNRAS, 318,1249

Bica, E. 1988, A\&A, 195, 76

Bica, E., \& Alloin, D. 1986a, A\&A, 162, 21

Bica, E., \& Alloin, D. 1986b, A\&AS, 66, 171

Bica, E., Dottori, H., \& Pastoriza, M. G. 1986c, A\&A, 156, 261

Bica, E., Alloin, D., \& Santos, Jr., J. F. C. 1990, A\&A, 235, 103

Cassatella, A., Barbero, J., Brocato, E., \& Geyer, E. H. 1996, A\&A, 306, 125

Da Costa, G. S., \& Hatzidimitriou, D. 1998, AJ, 115, 1934

de Oliveira, M. R., Dutra, C. M., Bica, E., \& Dottori, H. 2000, A\&AS, 146,57

Dutra, C. M., Bica, E., Clariá, J. J., \& Piatti, A. E. 1998, MNRAS, 305,373

Hodge, P. W., \& Wright, F. W. 1974, AJ, 79, 858

Holland, S., Fahlman, G. G., \& Richer, H. B. 1997, AJ, 114, 1488
Kron, G. E. 1956, PASP, 68, 125

Lauberts, A. 1982, The ESO/Uppsala Survey of the ESO (B) Atlas (Garching: European Southern Observatory)

Lindsay, E. M. 1958, MNRAS, 118, 172

Mighell, K. J., Sarajedini, A., \& French, R. S. 1998, AJ, 116, 2395

Olszewski, E. W., Suntzeff, N. B., \& Mateo, M. 1996, ARA\&A, 34, 511

Ostrov, P., Forte, J. C., \& Geisler, D. 1998, AJ, 116, 2854

Piatti, A. E., Clariá, J. J., \& Bica, E. 1999, MNRAS, 303, 65

Piatti, A. E., Santos Jr., J. F. C., Clariá, et al. 2001, MNRAS, 325, 792

Piatti, A. E., Bica, E., Clariá, J. J., Santos Jr., J. F. C., \& Ahumada, A. V. 2002, MNRAS, 335, 233

Pietrzyński, G., \& Udalski, A. 1999, Acta Astron., 49, 157

Rich, R. M., Shara, M., Fall, S. M., \& Zurek, D. 2000, AJ, 119, 197

Santos Jr., J.F.C., \& Bica, E. 1993, MNRAS, 260, 915

Santos Jr., J. F. C., Bica, E., Clariá, et al. 1995, MNRAS, 276, 1155

Sarajedini, A., Geisler, D., Shommer, R., \& Harding, P. 2000, AJ, 120, 2437

Schmidt, A. 1988, SPEED Users's Manual, Univ. Federal do Santa María, Brazil

Seaton, M. J. 1979, MNRAS, 187, 73p

Shara, M., Fall, S., Rich, M. R., \& Zurek, D. 1990, ApJ, 508, 570

Stone, R., \& Baldwin, J. 1983, MNRAS, 204, 347

van den Bergh, S. 1981, A\&AS, 46, 79

Westerlund, B. E., \& Glaspey, J. 1971, A\&A, 10, 1 